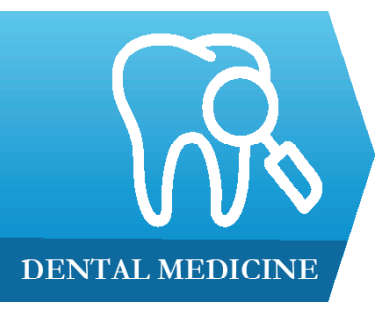

DENTAL MEDICINE

1) Department of Orthodontics and Dentofacial Orthopedics, Sri Aurobindo College of Dentistry, Indore (M.P.), 452001, India

2) National Oral Health Programme, Centre for Dental Education and Research, AIIMS, New Delhi, India

DOI: $10.15386 / \mathrm{mpr}-1132$

Manuscript received: 07.08 .2018

Received in revised form: 02.11.2018

Accepted: 17.11.2018

Address for correspondence:

rohit2111988@gmail.com

\title{
The effect of tooth morphology and vertical bracket positioning on resultant stress in periodontal ligament - a three dimensional finite element study
}

Rohit Kumar Maheshwari ${ }^{1}$, Ashish Garg ${ }^{1}$, Bhavna Virang ${ }^{1}$, Upendra Singh Bhadauria ${ }^{2}$

\begin{abstract}
Introduction. The purpose of this study is to evaluate the effect of change in vertical placement of bracket and effect of tooth morphology on stress developed on periodontal ligament with the help of three dimensional finite element modeling.

Methods. A three-dimensional model of the maxillary bone, maxillary right central incisor, lateral incisor and canine was designed based on the average dimensions of the anatomy and morphology given by Wheeler's and standard edgewise bracket with Slot of $0.022^{\prime \prime}$ X 0.028" inch was designed using the finite element method. Brackets were placed on each tooth, on the mentioned labial surface at variable distances from the cusp tip, and a full size archwire was virtually engaged into the bracket, then optimum orthodontic load of $2 \mathrm{~N}$ is applied and PDL stress were calculated.
\end{abstract}

Results. The lowest stress values were measured as bracket position changes from crest of teeth to the apical direction. By displacing the bracket gingivally from 1.5 to $6 \mathrm{~mm}$, a $16.2 \%$ decrease in stress level for central incisor, for lateral incisor the stress level decrease by $25.8 \%$ and for canine the stress level decrease by $21.6 \%$ thus our study confirms that variation in vertical bracket position results in change in resultant stress in PDL.

Conclusion. It can be concluded that the variation in the vertical position of the bracket on different tooth can have an important effect on the stresses developed in the PDL.

Keywords: FEM, vertical bracket position, PDL stress

\section{Introduction}

The edgewise system based on a 3-dimensional tooth control obtained by engaging a rectangular wire into a bracket with a rectangular slot was introduced by Angle [1]. The difficulties in obtaining satisfactory treatment with the standard edgewise technique resulted in the development of brackets with built-in torque in the early 1960's. Ten years later, the straight wire appliance (SWA) was introduced by Andrews. Taking advantage of the control offered by the edgewise system, he advocated the use of the straight wire appliance (SWA) [2]which greatly reduced the amount of routine wire bending needed for an average patient. Considering that SWA brackets contain the necessary information for the desired tooth position the only variables that still remain are tooth morphology and the position of the bracket on the surface of the tooth.

In the preadjusted appliance system, there is a different tip and torque value for every tooth. In order to do the effective comparison of stress changed between maxillary central incisor, lateral incisor and maxillary canine, the present study utilized standard edgewise bracket (no inbuilt tip and torque) with $2 \mathrm{~N}$ of optimum orthodontic load with the only variable being tooth morphology and vertical bracket height.

The Standard Edgewise bracket with slots of 0.022 " X 0.028 " inch were placed on each tooth, on the labial surface at variable distances from the cusp tip, and a full size archwire was virtually engaged into the bracket. The optimum orthodontic load of $2 \mathrm{~N}$ was applied and PDL stresses were calculated.

An analysis of the previous literature revealed that numerical methods 
to calculate stress and strain in the periodontium have been extensively used. Andersen KL, Pedersen EH and Melsen $\mathrm{B}$ [3] reported a marked variation in the stress distribution from cervix to apex when tipping forces were applied. The bodily movement of the tooth produced almost uniform stress distribution whereas the torquing movements showed stress patterns exactly opposite to that of tipping. Masticatory forces alone caused patterns similar to those achieved by combination of masticatory and orthodontic forces.

Few experimental studies reported that using the finite element method will be the best non-invasive method for studying this phenomenon of variation in bracket position and its relation to stress pattern.

FEM offers an ideal method for accurate modeling of the tooth and periodontium with its complicated3-dimensional geometry. It is comparatively more advantageous than other conventional methods for conducting studies as it generates a three-dimensional model with the freedom to simulate and study orthodontic force systems [4].Thus, taking into consideration the numerous advantages which FEM offers, it was used in the present study.

In previous studies $[5,6]$ the stresses and strains were investigated within the periodontal ligament and surrounding bone, consequent to orthodontic loading of a tooth by application of the finite element method. In the finite element model, bone was found to experience a low strain of $1 \times 10-5$, whereas the periodontal ligament experienced a strain of 0.1 with the "tooth model". This later figure above the threshold is usually reported to be necessary to initiate the remodeling process. Further developments in this rapidly advancing area of biomechanical research should facilitate a greater increase in our knowledge of tissue stress and strain after loading $[7,8]$.

Similarly, some authors developed finite element models to simulate dynamic orthodontic treatments of the translation, inclination, and rotation of the left mandibular canine with transparent tooth correction system. Piecewise static simulations were performed to replicate the dynamic process of orthodontic treatments. The distribution and change trends of canine's displacements and stresses in the canine's PDL during the three types of tooth movements were obtained. Canine's movement type significantly influences the distribution of canine's displacement and stresses in the canine's PDL. Changes in canine's displacement and stresses in the canine's PDL were exponential in transparent tooth correction treatment $[9,10]$.

Hence the present three dimensional finite element study was aimed to evaluate the effect of tooth morphology and vertical positioning on resultant stress in periodontal ligament. The objectives were to determine the effect of variation in vertical bracket position on the stress distribution in periodontal ligament of maxillary anteriors due to variation in vertical bracket position and to determine the effect of variation in tooth morphology on stress in maxillary anteriors. This evaluation will further help the orthodontist to learn the need for correct bracket positioning for better control on three dimensional tooth movements and for better treatment outcome.

\section{Methods}

The present study was done to evaluate the changed stress distribution in PDL with change in bracket position. In the present study, the three-dimensional model of the maxillary right central incisor, lateral incisor and canine were taken to evaluate the changed stress distribution in PDL with change in vertical bracket position.

The aim of the present study warranted the requirement to generate quality $3 \mathrm{D}$ mesh which necessitated the use of finite element software with good generation and meshing facility for accurate stress analysis. Hence, the present study utilized Solidworks 2016 (Dassault System Solidworks Corp. Massachusetts, U.S.A), Pro Engineering (PTC) and ANSYS 16.0 (Ansys, Inc Pennysylvania, United States) for the study in India.

The current study was divided under 5 heads [11]:

1) Construction of geometric models

2) Preparing the finite element mesh

3) Validation of model

4) Application of forces and boundary conditions

5) Analysis of stress pattern

1) Construction of geometric models

It was carried out in following steps:

A. Modeling of maxillary body (cortical and cancellous bone).

B. Modeling of central incisor, lateral incisor and canine.

C. Modeling of periodontal ligament.

D. Modeling of the Bracket and Wire.

Owing to the complexity of geometry curves of maxillary bone and teeth, in generating an accurate geometry, a section of bone block of definite dimensions was designed as shown in table I using the finite element method .This model included teeth, periodontal ligament (PDL), compact and cancellous bones.

Table I. Measurement of the teeth: All values are in $\mathrm{mm}$.

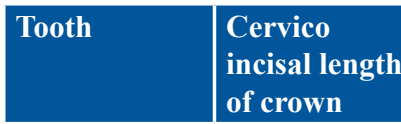

Central incisor

Lateral incisor

Canine

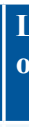

Mesio distal
diameter of
crown

Mesio distal
diameter of
crown at cervix

Labio lingual
diameter of
crown

Labio lingual
diameter at
cervix

Curvature
of cervical
line mesial

3.5

3.0

2.5
Curvature

of cervical line distal
6.5

7.5
5.0

5.5
6.0

8.0
5.0

7.0

5




\section{Solid models of each component}

\section{A. Modeling of maxillary body}

As cortical bone and lamina dura have the same mechanical properties, both were modeled as cortical bone (Figure 1).

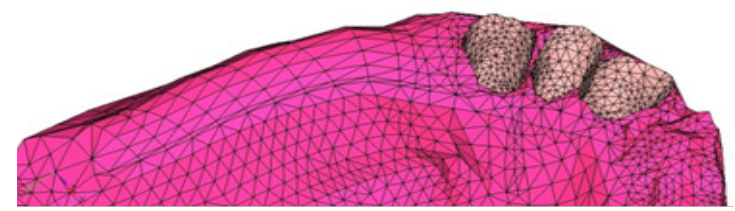

Figure 1. Meshing of maxillary bone with alveolar socket containing Pdl.

\section{B. Modeling of central incisor, lateral incisor and canine}

Due to similar mechanical properties of the dentine and cementum, they were both considered as root dentine. To simplify the model in this study, only the mechanical properties of dentine were used [12]. To determine the dimensions of the tooth, labial surface curvature, crown height, root length, buccolingual width, and mesiodistal width of the crown were designed.

To design the tooth model initially, the labial, palatal, distal, and mesial views of the given tooth were taken by scanner and converted into JPG files. The files were subsequently imported into AutoCAD and the boundary lines were plotted via spline curves. The plotted DWG files were generated and imported into solid work to produce three-dimensional volumetric models of the tooth's components. After the completion of volumetric model, the output X-T file was imported into ANSYS (Figure 2).

As the maxillary central incisor, lateral incisor and canine undergo the most detailed tooth movement and are at the higher risk for root resorption they were selected in the present study [13].

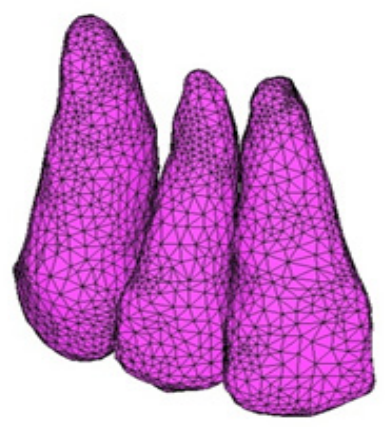

Figure 2. Showing FEM meshing of maxillary right central incisor, lateral incisor and canine.

\section{Modeling of periodontal ligament}

PDL was designed with a uniform thickness of 0.2 $\mathrm{mm}$ as described by Coolidgeand an isotropic behavior to PDL was assigned (Figure 3) [14].

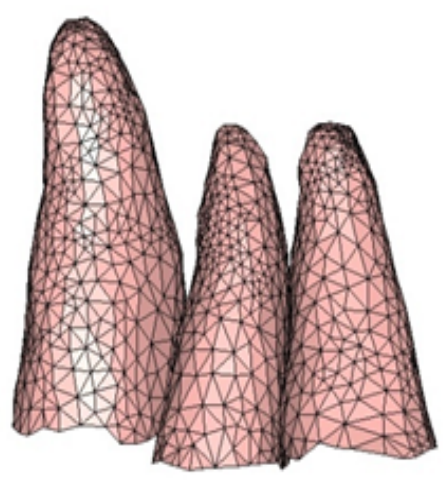

Figure 3. Meshing of periodontal ligament of maxillary right central incisor, lateral incisor and canine.

\section{Modeling of the bracket and wire}

A bondable stainless steel twin metal bracket of standard edgewise from DENTSPLY was designed, incorporating 0.022 " X 0.028 " inch bracket slot (Figure 4). The bracket was 'attached' to the tooth surface, and composite of thickness $0.2 \mathrm{~mm}$ [15] was added to fill the gaps between the bracket and the tooth. A 0.019 " X 0.025 " inch stainless steel wire was designed and engaged into the brackets (Figure 4).

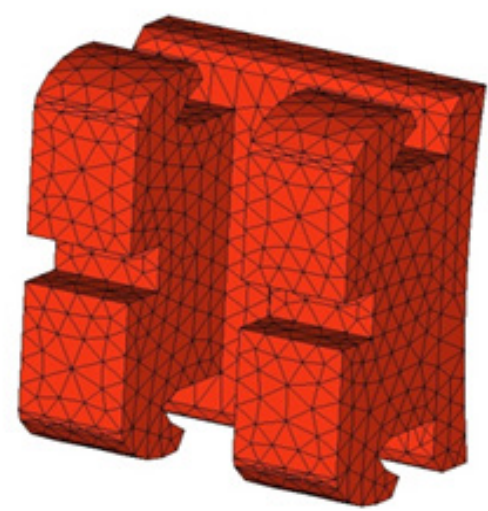

Figure 4. Meshing of bracket.

\section{2) Preparing the finite element mesh Meshing of all models}

In the present study, four nodded tetrahedral elements model was used and consisted of 101816 of elements and 22381 nodes (Figure 5). To simplify the model and reduce the analysis time all materials were assumed to be isotropic and elastic. The mechanical properties (Poisson's ratio, Young's modulus) of the periodontal ligament, tooth, cortical and cancellous bone were obtained from previous studies given in Table II [16]. 


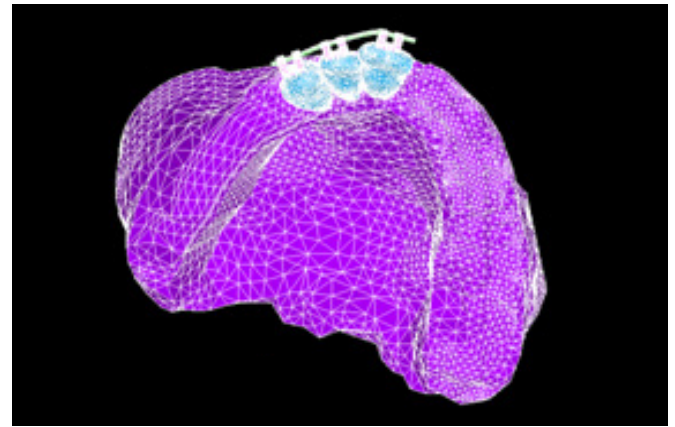

Figure 5. Meshing of complete model.

Table II. Mechanical properties of Structures [12].

\begin{tabular}{l|c|c|}
\hline Materials & Young's modulus (Mpa) & Poisson's ratio \\
\hline Enamel & 84,100 & 0.33 \\
Dentin & 18,600 & 0.30 \\
Alveolar bone & 490 & 0.30 \\
Cortical bone & 14,700 & 0.30 \\
PDL & 0.1 & 0.45 \\
Composite & 2,140 & 0.31 \\
Stainless steel & 200,000 & 0.30
\end{tabular}

\section{3) Validation of model}

Highlighted wrapped, distorted or skewed elements were removed. Models were viewed at different orientations to confirm the corrections of the models.

\section{4) Application of boundary conditions}

Boundary conditions were defined at all peripheral nodes of the bone with zero degree of movement in all directions in order to calculate stress in PDL.

\section{5) Analysis of stress pattern}

The computerized model was transferred to ANSYS software (ANSYS WB 16). All the previously mentioned conditions (material properties, interface condition, meshing and loading) were included in this software. The rectangular wire was placed into the bracket in complete contact with the walls of the slot and $2 \mathrm{~N}$ of load was applied on it.

\section{Stress in the PDL}

The Von-Mises stress in the PDL surface adjacent to

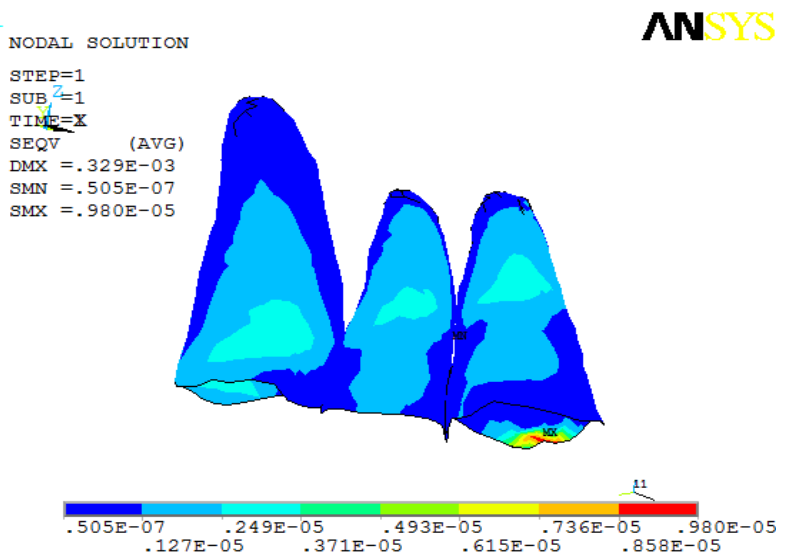

Figure 6. Stress calculated at $1.5 \mathrm{~mm}$ of vertical bracket position from crest of teeth with loading of $2 \mathrm{~N}$ of force. the roots was calculated for the various bracket distances.

\section{Tooth Morphology}

The labial contour of the crown surface differs at different heights on the crown of the same tooth. Therefore, tooth morphology was also considered in this study.

\section{Analysis variables}

The bracket's vertical position initiated at $1.5 \mathrm{~mm}$ (distance between the center of the slot and the cusp tip) was the most occlusal distance that provided a good fit between the bracket base and the tooth surface. The bracket was then displaced $4.5 \mathrm{~mm}$ gingivally in $0.5 \mathrm{~mm}$ increments between subsequent models.

\section{Statistical Analysis}

The data collected was entered in Microsoft Excel and subjected to statistical analysis using Statistical Package for Social Sciences (SPSS, IBM version 20.0). The level of significance was fixed at $5 \%$ and $p \leq 0.05$ was considered statistically significant. Descriptive statistics was used to determine the variables.

\section{Results}

Initial analysis was carried out with bracket distance from the crest of the teeth as $1.5 \mathrm{~mm}$. The boundary conditions remained same for all the models except the position of the bracket by which the moment values varies in the models. When the optimum force required for orthodontic tooth Movement of 2N [17] was applied at various vertical bracket position from the $1.5 \mathrm{~mm}$ to the $6 \mathrm{~mm}$, the important structural parameters of stresses were observed for different models (Figure 6 and Figure 7). We considered in the present study that the maximum Von-Mises stress resulted from different bracket positions. Figures 8 and 9 shows the maximum Von-Mises stress resulted by application of $2 \mathrm{~N}$ force. The graph revealed that the maximum Von-Mises stress was at the central incisor in changing bracket position from the $1.5 \mathrm{~mm}$ to the $6 \mathrm{~mm}$. Figures 3, 4 and 5 shows the different values of maximum von mises stress for cental incisor, lateral incisor and canine respectively at various vertical bracket position from the $1.5 \mathrm{~mm}$ to the $6 \mathrm{~mm}$.

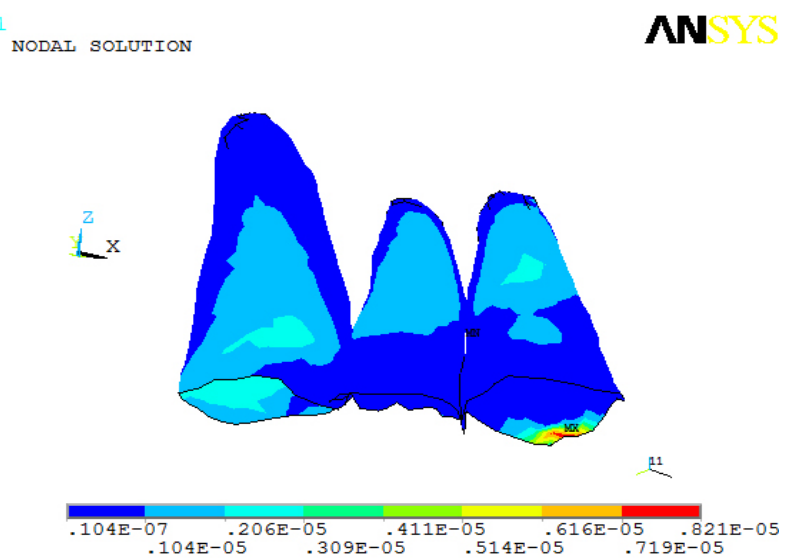

Figure 7. Stress calculated at $6 \mathrm{~mm}$ of vertical bracket position from crest of teeth with loading of $2 \mathrm{~N}$ of force 


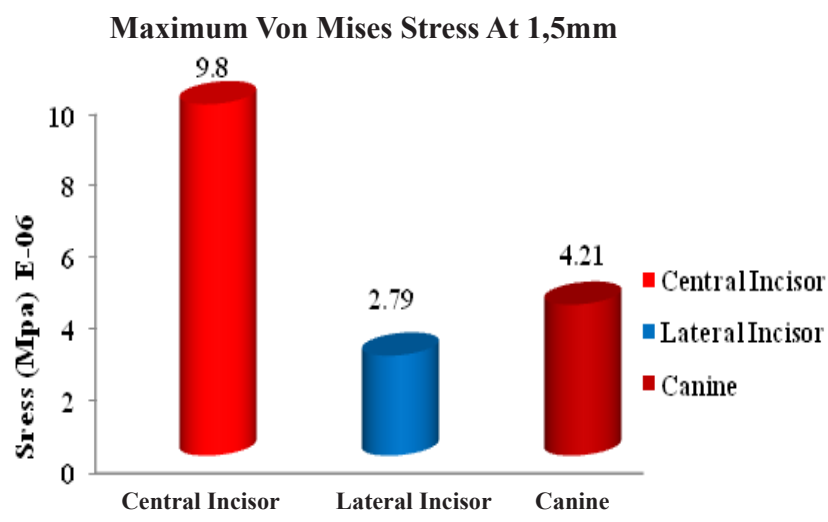

Figure 8. Maximum Von Mises stress at $1.5 \mathrm{~mm}$.

\section{Discussion}

In the present study, a maxillary central incisor, lateral incisor and canine were designed, incorporating labial surface curvature dimensions of the tooth including crown height, root length, buccolingual width, and mesiodistal width of the crown. Standard edgewise bracket with slot of 0.022 " X 0.028 " inch with zero degree torque was placed on each tooth, on labial surface at variable distances from the cusp tip, and a full size arch wire was virtually engaged into the bracket, optimum orthodontic load of $2 \mathrm{~N}$ was subsequently applied and the resultant PDL stress were calculated.

In orthodontics, the study of initial stress concentration in the PDL is important because the ligament acts as a mediator for the tooth movement and any alteration from the optimal force produces adverse effects on the tooth and periodontium such as apical root resorption and alveolar bone loss.

Orthodontic root resorption is frequently preceded by hyalinization of the PDL. Kurol and Owman-Moll [18] reported hyalinized areas opposite to an intact root surface or close to an area of root resorption. An optimal force intends to induce a maximal cellular response and establishes stability of the tissue. An unfavorable force does not result in a precise biologic response and may initiate adverse tissue reactions. Ankita Anil Ringane [19] reported Von Mises stresses in the bone and mini-implant were significantly higher in the lingual technique as compared to the labial technique. Middleton et al. [7] used FEM to evaluate stresses and strains within the PDL and surrounding bone consequently to orthodontic loading of a tooth. These results suggested that the remodeling process may be controlled by PDL rather than the bone. During the initial force application, compression in limited areas in PDL frequently impedes vascular circulation and cell differentiation causing degradation of cells and vascular structures rather than proliferation and differentiation. The tissue change during this period is called hyalinization. The process of hyalinization is dependent on the local morphology of the compressed area, the magnitude of the applied force on the tooth and the duration of this force.
Maximum Von Mises Stress At $6 \mathrm{~mm}$

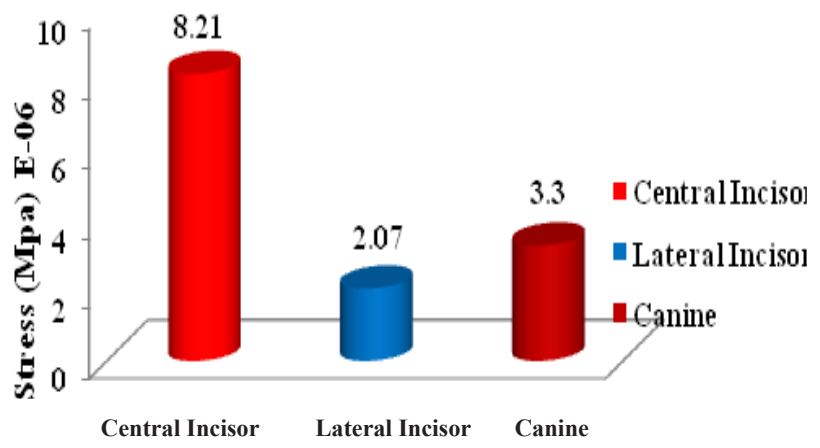

Figure 9. Maximum Von Mises stress at $6 \mathrm{~mm}$.

The most frequent complication preventing rapid tooth movement occurs when the applied force compresses the tooth against the alveolar bone that the PDL responds with local degeneration and sterile necrosis instead of cells that would have been able to perform the necessary reconstruction. This situation may lead to permanent damage on the involved tooth and its periodontium.

The present study highlights a method for in-vitro quantification of initial stresses and displacement perceived by tooth and periodontium during orthodontic tooth movement with change in vertical bracket position. In this study the highest stress were developed at the central incisor comparatively to lateral incisor and canine for the same bracket height and orthodontic load, which may be due to variation in tooth morphology. By displacing the bracket gingivally from 1.5 to $6 \mathrm{~mm}$, a $16.2 \%$ decrease in stress level for central incisor, $25.8 \%$ decrease for the lateral incisor and $21.6 \%$ decrease for canine was noted. Hence, our study confirms that variation in vertical bracket position result in change in resultant stress in PDL and also confirms that due to variation in bracket position the highest stress was developed in central incisor which is most susceptible tooth for root resorption. In our study the relatively highest stress was developed for central incisor which is line with the study conducted by Vikram et al. [20] and Jones et al [21]. Our study revealed that the cervical region of the root and the areas close to the alveolar crest are among the main areas of stress concentration which is in accordance with the study done by Sardarian et al [22].

In our study the highest compressive stress were developed in apical area where as the highest tensile stress was constricted at cervix of both incisors and canine which is in line with the study done by Shahri et al [12]. Papageorgiou et al. [23], in their study reported that Strains in the PDL were affected mainly by CRA (up to 54 per cent), followed by bracket positioning (up to 45 per cent). These results shine light on the importance of proper bracket positioning when using the straight wire appliance. However, the differences in the absolute values in the results obtained between the present study and those of the other previous studies could be due to the assumptions of physical properties of the tooth 
and periodontium, differences in morphometric of the tooth and PDL dimensions, the software used for the analysis and the number of nodes and elements used in the study.

The results of the present study offer encouraging clinical possibilities and must be accepted with guarded optimism. Further research is needed to compare the effect of tooth morphology and vertical bracket positioning on change on resultant stress in periodontal ligament.

\section{Conclusion}

The orthodontic forces applied during the tooth movement results in relatively high stress on the periodontal ligament. Based on the findings of the present study it can be concluded that the highest Von- Mises stress distribution was always around the cervical even at variable bracket position resulting in a more susceptible area for potential tissue damage. When an evaluation of stress distribution according to the teeth was done it was seen that the highest stress was developed at the central incisor when compared to lateral and canine. Future studies warrant attention to factors such as type of bracket slot, arch wire, the resin tooth and resin bracket interface.

\section{Acknowledgments}

I would like to thank Dr. M. L. Jain and Mr. Nagabhushana for assisting me with ANSYS software.

\section{References}

1. Angle EH. The latest and best in orthodontic mechanism. Dent Cosmos. 1928;70:1143-1158.

2. Andrews LF. The straight-wire appliance. Explained and compared. J Clin Orthod. 1976;10:174-195.

3. Andersen KL, Pedersen EH, Melsen B. Material parameters and stress profiles within the periodontal ligament. Am J Orthod Dentofacial Orthop. 1991;99:427-440.

4. Bobak V, Christiansen RL, Hollister SJ, Kohn DH. Stressrelated molar responses to the transpalatal arch: a finite element analysis. Am J Orthod Dentofacial Orthop. 1997;112:512-518.

5. Field C, Ichim I, Swain MV, Chan E, Darendeliler MA, $\mathrm{Li} \mathrm{W}$, et al. Mechanical responses to orthodontic loading: a 3-dimensional finite element multi-tooth model. Am J Orthod Dentofacial Orthop. 2009;135:174-181.

6. Cobo J, Argüelles J, Puente M, Vijande M. Dentoalveolar stress from bodily tooth movement at different levels of bone loss. Am J Orthod Dentofacial Orthop. 1996;110:256-262.

7. Middleton J, Jones M, Wilson A. The role of the periodontal ligament in bone modeling: the initial development of a timedependent finite element model. Am J Orthod Dentofacial Orthop. 1996;109:155-162.

8. Miethke RR, Melsen B. Effect of variation in tooth morphology and bracket position on first and third order correction with preadjusted appliances. Am J Orthod Dentofacial Orthop. 1999;116:329-335.

9. Cai Y, Yang X, Yao J. Finite element method analysis of the periodontal ligament in mandibular canine movement with transparent tooth correction treatment. BMC Oral Health. 2015;15:106.

10. Kumar JB, Reddy GJ, Sridhar M, Reddy TJ, Reddy PJ, Rao SS. A finite element analysis of initial stresses and displacements in the tooth and the periodontium in periodontally compromised simulations: Labial versus lingual force application. J NTR Univ Health Sci. 2016;5:34-43.

11. Logan, DL. A First Course in the Finite Element Method. 5th ed. Stamford: Cengage Learning; 2012: pp 72-141.

12. Shahri F, Fakour SR, Shahri F, Hashemzehi H. An investigation into stress distribution and determination of optimum force for torque movement on a tooth using the finite element method (FEM). RJPBCS. 2016;7:102-111.

13. Armstrong D, Shen G, Petocz P, Darendeliler MA. A comparison of accuracy in bracket positioning between two techniques--localizing the centre of the clinical crown and measuring the distance from the incisal edge. Eur J Orthod. 2007;29:430-436.

14. Coolidge ED. The thickness of the human periodontal membrane. J Am Dent Assoc Dent Cosm. 1937;24:1260-1270.

15. Papageorgiou SN, Keilig L, Hasan I, Jäger A, Bourauel C. Effect of material variation on the biomechanical behaviour of orthodontic fixed appliances: a finite element analysis. Eur J Orthod. 2016;38:300-307.

16. Liao Z, Chen J, Li W, Darendeliler MA, Swain M, Li Q. Biomechanical investigation into the role of the periodontal ligament in optimising orthodontic force: a finite element case study. Arch Oral Biol. 2016;66:98-107.

17. Kojima Y, Fukui H. A finite element simulation of initial movement, orthodontic movement, and the center of resistance of the maxillary teeth connected with an archwire. Eur J Orthod. 2014;36:255-261.

18. Kurol J, Owman-Moll P. Hyalinization and root resorption during early orthodontic tooth movement in adolescents. Angle Orthod.1998;68:161-165.

19. Ringane AA, Hattarki R. Comparison of stress distribution on bone and mini-implants during en-masse retraction of maxillary anterior teeth in labial and lingual orthodontics: A three-dimensional finite element analysis. Indian J Health Sci Biomed Res. 2018;11:130-135.

20. Vikram NR, Senthil Kumar KS, Nagachandran KS, Hashir YM. Apical stress distribution on maxillary central incisor during various orthodontic tooth movements by varying cemental and two different periodontal ligament thicknesses: a FEM study. Indian J Dent Res. 2012;23:213-220.

21. Jones ML, Hickman J, Middleton J, Knox J, Volp C. A validated finite element method study of orthodontic tooth movement in the human subject. J Orthod. 2001;28:29-38.

22. Sardarian A, Danaei S, Shahidi S, Boushehri SG, Geramy A. The effect of vertical bracket positioning on torque and the resultant stress in the periodontal ligament--a finite element study. Prog Orthod. 2014;15:50.

23. Papageorgiou SN, Sifakakis I, Keilig L, Patcas R, Affolter $\mathrm{S}$, Eliades $\mathrm{T}$, et al. Torque differences according to tooth morphology and bracket placement: a finiteelement study. Eur J Orthod. 2017;39:411-418. 